\title{
Manifestações respiratórias e doenças esofágicas
}

\author{
Respiratory manifestations and esophageal diseases
}

\section{Elie Fiss}

Das doenças esofágicas relacionadas às manifestações respiratórias, a doença do refluxo gastroesofágico (DRGE) é, sem dúvida, a que mais se apresenta no nosso cotidiano.

A DRGE pode estar associada à asma e à fibrose pulmonar. Além disso, a sintomatologia de tosse crônica é uma das suas mais freqüentes manifestações clínicas e, conseqüentemente, este é um dos principais sintomas que levam um paciente à procura de uma consulta médica. ${ }^{(1)}$

Alguns autores relataram a elevada incidência de sintomas digestivos, tais como pirose, disfagia, engasgos e regurgitação, em pacientes asmáticos. Estes sintomas variam entre $24 \%$ a $89 \%$, dependendo do estudo. ${ }^{(2-8)} 0$ contrário também é válido, pois há pacientes com doença esofágica cuja única manifestação clinica é respiratória, o que passa a ser um problema na investigação diagnóstica, especialmente para o pneumologista.

Para alguns autores, até 30\% dos pacientes com tosse crônica desencadeada por DRGE podem apresentar a tosse como a única manifestação clinica da doença esofágica.

A associação entre asma e DRGE tem sido muito estudada, e alguns mecanismos são sugeridos para explicar esta relação. ${ }^{(2-8)}$ Microaspiração de suco gástrico e reflexo esôfago-brônquico, provavelmente com mediação vagal, são os mais citados pela literatura quanto às manifestações respiratórias. ${ }^{(3-6)} \mathrm{A}$ instilação de acido clorídrico $(0,1 \mathrm{~N})$ no esôfago distal desencadeou uma redução do volume expiratório forçado no primeiro segundo $\left(\mathrm{VEF}_{1}\right)$ maior que $20 \%$ em alguns pacientes asmáticos. ${ }^{(3)}$ Em outros estudos, a instilação de soro fisiológico e a própria sondagem esofágica foram capazes de desencadear uma redução de $\mathrm{VEF}_{1}$, mas sempre menor que a causada pelo ácido clorídrico. ${ }^{(6)}$ A presença de refluxo gastroesofágico (RGE) proximal foi muito associada à dor torácica, ao passo que a presença de RGE distal foi associada à tosse crônica. ${ }^{(3)}$

Por outro lado, a própria asma pode desencadear uma disfunção vagal, além de alterar as pressões do gradiente tóraco-abdominal, favorecendo o aparecimento de hérnia hiatal e RGE. Além disso, o uso prolongado de broncodilatadores resulta em uma redução da pressão do esfíncter inferior do esôfago, e os corticóides podem aumentar o tempo de exposição ácida do esôfago. 0 resultado final desta relação é o aumento da hiper-reatividade brônquica em indivíduos com DRGE, provavelmente pela liberação de peptídeos inflamatórios ou por reflexo vagal. Em um estudo anterior, pudemos mostrar exatamente a hiper-reatividade brônquica em pacientes com DRGE sem doença pulmonar associada. $^{(4)}$

Apesar de todos os estudos já publicados, ainda existem muitas controvérsias sobre os mecanismos que fazem com que manifestações respiratórias tenham origem em doenças esofágicas e vice-versa, se o tratamento das doenças esofágicas pode controlar as doenças respiratórias e, em caso afirmativo, de que maneira.

Em estudos nos quais realizou-se o tratamento clínico ou cirúrgico da DRGE, obteve-se melhora de cerca de 70\% dos sintomas de asma, com redução dos medicamentos; houve também melhora da função pulmonar, mas em menor escala (25\%). Em contrapartida, outros autores não acharam benefícios consistentes na asma tratada com a associação de medicamentos para DRGE. ${ }^{(8)}$

Como podemos ver, ainda são necessários muitos estudos, talvez com maior casuística ou maior acompanhamento das terapias aplicadas. Para aumentar ainda mais o campo da pesquisa da associação entre RGE e doenças respiratórias, não podemos esquecer a síndrome da apnéia do sono, cuja relação já tem sido estudada por diversos autores, e a doença pulmonar obstrutiva crônica, que nos preocupa cada vez mais pela sua incidência e prevalência, e que ocorre em um número elevado de pacientes portadores de DRGE. ${ }^{(7)}$

Diante do exposto, qual seria a relação entre estas doenças, de causa e conseqüência ou seriam doenças coexistentes? Provavelmente as duas respostas estão corretas dependendo da variabilidade individual.

0 pneumologista deve, em minha opinião, em algum momento investigar a presença de doenças esofágicas nos pacientes com manifestações respiratórias, independentemente da presença ou não de sintomas digestivos.

$\mathrm{Na}$ rotina, sem dúvida nenhuma, o exame subsidiário mais solicitado com esta finalidade é a endoscopia digestiva, provavelmente pela facilidade de acesso, mas devemos saber que é um método que tem limitações para o diagnóstico da DRGE. ${ }^{(5)} \mathrm{A}$ pHmetria esofágica de $24 \mathrm{~h}$ e, mais recentemente, a impedanciometria seguem sendo o padrão ouro para o diagnóstico de DRGE. Entretanto, elas também sofrem críticas quanto a sua interpretação, tais como a modificação do hábito alimentar durante a realização do exame, o não reconhecimento do refluxo básico pela 
pHmetria e principalmente a falta de acesso para sua execução em todos os centros.

$\mathrm{Na}$ presente edição do Jornal Brasileiro de Pneumologia, há um estudo de Machado et al. que mostra exatamente esta associação entre os dois sistemas. ${ }^{(9)}$ Foram 1.170 pacientes com manifestações respiratórias avaliados através de pHmetria de $24 \mathrm{~h}$ e esofagomanometria. 0 estudo mostrou exatamente a necessidade da avaliação independentemente dos sintomas, uma vez que, por exemplo, $35,2 \%$ dos pacientes asmáticos com RGE patológico não apresentavam nenhuma manifestação digestiva.

A DRGE esteve presente entre 39\% e $44 \%$ dos pacientes, dependendo da doença respiratória, com especial destaque para a asma e sua associação com a hipotonia do esfíncter inferior do esôfago. Os autores sugerem, como conclusão, que a DRGE pode ser considerada uma causa extrapulmonar de sintomas respiratórios crônicos.

Muitos são ainda os pontos a serem elucidados em relação às doenças respiratórias e as doenças esofágicas: mecanismos fisiopatológicos; métodos diagnósticos e seus parâmetros de normalidade; identificação dos pacientes que se beneficiam do tratamento duplo e qual motivo os diferenciam; e melhores opções terapêuticas e quando indicá-las. Usei principalmente referências do nosso Jornal Brasileiro de Pneumologia, pois aos poucos estamos construindo um grande conhecimento sobre este assunto, com indiscutível qualidade técnica. Tudo isso em função dos melhores resultados para nossos pacientes.

\section{Elie Fiss \\ Professor Titular de Pneumologia da Faculdade de Medicina do ABC - FMABC - Santo André, São Paulo}

\section{Referências}

1. Gurski RR, Rosa AR, Valle E, Borba MA, Valiati AA. Extraesophageal manifestations of gastroesophageal reflux disease. J Bras Pneumol. 2006;32(2):150-60.

2. Araujo AC, Ferraz E, Borges MC, Terra-Filho J, Vianna EO. Investigation of factors associated with difficult-to-control asthma. J Bras Pneumol. 2007;33(5):495-501.

3. Fiss E. Estudo do reflexo esôfago brônquico em pacientes asmáticos [doctoral thesis]. São Paulo: Faculdade de Medicina da Universidade de São Paulo; 1993.

4. Lapa MS, Rodrigues R, Fiss, E. Bronchial hyperreactivity in patients with gastroesophageal reflux disease. J Bras Pneumol. 2007;31(4):286-91.

5. Moraes Filho JPP, Rodrigues TM, Fiss, E. Asma e refluxo gastroesofágico. ABCD. Arquivos Brasileiros de Cirurgia Digestiva. 1992;7(p. supl 1).

6. Araújo AC, Aprile LR, Terra-Filho J, Dantas RO, Martins MA, Vianna EO. The effect of esophageal acidification on bronchial obstruction in individuals with asthma with gastroesophageal reflux. J Bras Pneumol. 2005;31(5):13-9.

7. Fortunato GA, Machado MM, Andrade CF, Felicetti C, Camargo JJ, Cardoso PF. Prevalence of gastroesophageal reflux in lung transplant candidates with advanced lung disease. J Bras Pneumol. 2008;34(10):772-8.

8. Martins, MA. Asthma and gastroesophageal reflux disease. J Bras Pneumol. 2007;33(2):xi-xii.

9. Machado MM, Cardoso PF, Ribeiro 10, Zamin 1 Jr, Eilers RJ. Esophageal manometry and 24-h esophageal pH-metry in patients with respiratory symptoms. J Bras Pneumol. 2008;34(12):1040-8. 